\title{
The Relationship between Serum Endocan Levels and Depression in Alzheimer's Disease
}

\author{
Kyung Hee Yoon, ${ }^{1,2}$ So Yeon Kim, ${ }^{1}$ Yoo Sun Moon, ${ }^{1,2}$ Daeyoung Roh, ${ }^{1}$ \\ Sang Kyu Lee, ${ }^{1}$ and Do Hoon Kim ${ }^{1}$ \\ ${ }^{1}$ Department of Psychiatry, Chuncheon Sacred Heart Hospital, Hallym University College of Medicine, \\ Chuncheon 200-704, Republic of Korea \\ ${ }^{2}$ Institute for Skeletal Aging, Chuncheon Sacred Heart Hospital, Hallym University College of Medicine, \\ Chuncheon 200-704, Republic of Korea
}

Correspondence should be addressed to Do Hoon Kim; dhkim0824@gmail.com

Received 17 November 2015; Accepted 24 December 2015

Academic Editor: George Perry

Copyright (C) 2016 Kyung Hee Yoon et al. This is an open access article distributed under the Creative Commons Attribution License, which permits unrestricted use, distribution, and reproduction in any medium, provided the original work is properly cited.

Objectives. Growing evidence suggests that angiogenic vascular factors may be involved in the pathogenic mechanism of Alzheimer's disease $(\mathrm{AD})$, and recently endocan has been proposed as an angiogenic biomarker. The aim of this study was to measure serum endocan levels according to the presence of depression in $\mathrm{AD}$ and to investigate the association among the serum endocan levels, cognitive function, and depression in these patients. Methods. Serum endocan levels were measured in 26 AD patients with depression, $29 \mathrm{AD}$ patients without depression, and 29 healthy controls using an enzyme-linked immunosorbent assay kit. The Mini-Mental State Examination-Korean version (MMSE-KC) and the Korean version of the Geriatric Depression Scale-Short Form (SGDS-K) were used to evaluate cognitive function and depressive symptoms, respectively. Results. Serum endocan levels were significantly lower in $\mathrm{AD}$ patients with depression than in $\mathrm{AD}$ patients without depression or healthy controls. Serum endocan levels were negatively correlated with SGDS-K scores but not with MMSE-KC scores in AD patients. Conclusions. This study suggests that serum endocan levels might be associated with depression in AD. Future studies are needed to investigate the pathophysiological mechanisms or the role of endocan in $\mathrm{AD}$ with depression.

\section{Introduction}

Although amyloid plaques, among other amyloid peptide fragments, have been identified as the primary pathological lesions in Alzheimer's disease (AD), it is not clear how these plaques are formed in the brain. It has been reported that angiogenic activation of the brain endothelium in AD leads to deposition of $\beta$-amyloid plaques and neurotoxic peptides are then secreted, which kill cortical neurons [1]. Growing evidence suggests that angiogenic vascular factors may be involved in the pathogenic mechanism of $\mathrm{AD}$ [1-3]. A number of studies have measured vascular endothelial growth factor (VEGF) levels in AD patients, but their results were inconsistent [3-5]. Recently, we found that serum VEGF levels were significantly increased in AD patients who had comorbid depression, which indicates that VEGF may be associated with depression in $\mathrm{AD}[6]$.

Interestingly, genetic expression of endocan is regulated by VEGF $[7,8]$, and endocan has also been proposed as a marker of angiogenesis $[9,10]$. Endocan is a novel molecule which is specific to human endothelial cells. A number of studies have shown that endocan is overexpressed in multiple types of cancers $[8,11-17]$, which suggests that endocan could be involved in angiogenesis in various types of tumors and tumor growth. Moreover, increasing evidence has recently indicated that endocan may play a role in endotheliumdependent pathological disorders [18-23] and inflammatory diseases [24-28]. These findings suggest that endocan may be involved in endothelial dysfunction and inflammatory pathology. 
We consider that endocan is possibly related to the pathophysiology of AD. However, no previous study has investigated the serum endocan levels in $\mathrm{AD}$ or any possible association between these levels and depression in $\mathrm{AD}$. Therefore, we measured the serum endocan levels in $\mathrm{AD}$ patients with and without depression and attempted to verify the association among the serum endocan levels, cognitive function, and depression.

\section{Methods}

2.1. Subjects and Procedures. The subjects were recruited from the Dementia Clinic of Chuncheon Sacred Heart Hospital. The diagnosis of AD was based on the Diagnostic and Statistical Manual of Mental Disorders, Fourth Edition (DSM-IV) and neuropsychological tests that used the Korean version of the Consortium to Establish a Registry for Alzheimer's Disease Assessment Packet (CERAD-K). The diagnosis of depression in $\mathrm{AD}$ patients was based on the criteria proposed by Olin et al. [29] who developed a scale to identify the presence of depression in $\mathrm{AD}$ more precisely than the previously used DSM-IV criteria.

The subjects comprised $26 \mathrm{AD}$ patients with depression (AD + depression), $29 \mathrm{AD}$ patients without depression (AD depression), and 29 healthy controls, all more than 65 years of age. Study protocols were reviewed and approved by the Ethics and Medical Research Committee of Chuncheon Sacred Heart Hospital, and all subjects gave written informed consent.

We excluded patients with inflammatory disease, malignancy, vascular dementia, other neurodegenerative diseases, diabetes mellitus, atherosclerosis, fracture, or history of substance abuse and patients who were taking medicines that contained acetylsalicylic acid or 5 alpha-reductase inhibitors, which are known to influence serum angiogenic factors. None of the subjects were taking any medicines except for antihypertensive agents. Current smoking status was established, and height and body weight were measured to calculate body mass index.

\subsection{Measures}

2.2.1. Neuropsychological Assessment. The subjects were examined by a trained neuropsychologist. The Mini-Mental State Examination in the Korean version of CERAD Assessment Packet (MMSE-KC) [30] was used to assess cognitive function; the scale ranges from 0 to 30 , and higher scores indicate better cognitive function. AD severity was measured with the Clinical Dementia Rating (CDR) [31], where CDR $0=$ no dementia, CDR $0.5=$ questionable dementia, $\mathrm{CDR}$ $1=$ mild dementia, $\mathrm{CDR} 2=$ moderate dementia, and CDR 3 = severe dementia, respectively. Depression severity was measured with the Korean version of the Geriatric Depression Scale-Short Form (SGDS-K) [32], which consists of 15 yes-or-no items about depressive symptoms; scores range from 0 to 15 , with a higher score indicating more severe depression.
2.2.2. Serum Endocan. Blood samples were drawn from an antecubital vein in a sitting position under nonfasting conditions. Samples were stored in plain tubes without ethylenediaminetetraacetic acid (EDTA) and were centrifuged at $1610 \mathrm{~g}$ for $10 \mathrm{~min}$ at $4^{\circ} \mathrm{C}$. In order to reduce any technical or personal bias, the sampling was performed by two welltrained medical technologists in a standardized manner. The samples were collected during the study and stored at $-80^{\circ} \mathrm{C}$ until use, for a maximum time period of 1 year. Serum endocan levels were measured with an enzyme-linked immunosorbent assay kit (ELISA; LUNGINNOV Systems, Lille, France). The assay range of the Endocan ELISA kit was $0.3-10 \mathrm{ng} / \mathrm{mL}$. Intra-assay and interassay coefficients of variation for the endocan assay were $<4.40 \%$ and $<7.59 \%$, respectively. The limit of quantification for the assay was $0.3 \mathrm{ng} / \mathrm{mL}$.

2.3. Statistical Analysis. Demographics and clinical data were compared among the groups using one-way analysis of variance (ANOVA) and chi-square tests. Differences in serum endocan levels among the groups were measured using ANOVA, and Duncan's test was used for the post hoc analysis. Pearson's correlation analyses were performed between serum endocan levels and SGDS-K and MMSEKC scores. Statistical analyses were performed using the Statistical Package for the Social Sciences (IBM, SPSS version 20.0). $p<0.05$ was considered statistically significant.

\section{Results}

3.1. Demographic Characteristics and Clinical Data. The demographic characteristics and clinical data of the study population are presented in Table 1 . There were no significant differences among the groups with respect to age, sex distribution, hypertension, body mass index, or smoking status. The mean MMSE-KC scores were significantly different; specifically, the mean score was lower in $\mathrm{AD}$ patients than in healthy controls $(F(2,81)=166.00, p=0.000)$. The mean CDR score was significantly higher in AD patients than in healthy controls $(F(2,81)=61.32, p=0.000)$. The mean SGDS-K score was significantly higher in the $\mathrm{AD}+$ depression group than in either the $\mathrm{AD}$ - depression group or healthy controls $(F(2,78)=111.71, p=0.000)$.

3.2. Serum Endocan Levels. Serum endocan levels were significantly lower in the $\mathrm{AD}+$ depression group compared with the $\mathrm{AD}$ - depression group and healthy control group; levels in each group are shown in Figure 1. Endocan levels in the $\mathrm{AD}+$ depression, $\mathrm{AD}$ - depression, and healthy control groups were $0.51 \pm 0.13,0.70 \pm 0.18$, and $0.64 \pm 0.20 \mathrm{ng} / \mathrm{mL}$ (mean $\pm \mathrm{SD}$ ), respectively. Post hoc comparisons of endocan levels indicated that these levels were lower in the $\mathrm{AD}+$ depression group than in the $\mathrm{AD}$ - depression group and the healthy controls $(F(2,81)=7.57, p=0.001)$.

3.3. Correlations among Serum Endocan Levels, Depressive Symptoms, and Cognitive Function Scores. Endocan levels were negatively correlated with SGDS-K scores in $\mathrm{AD}$ 
TABLE 1: Demographic characteristics and clinical data of study subjects.

\begin{tabular}{|c|c|c|c|c|c|}
\hline Variables & $\mathrm{AD}+$ depression $(n=26)$ & $\mathrm{AD}-$ depression $(n=29)$ & Control $(n=29)$ & Statistics & $p$ \\
\hline Sex (male/female) & $4 / 22$ & $7 / 22$ & $11 / 18$ & $\chi^{2}=3.70$ & 0.157 \\
\hline Age $($ mean $\pm S D)$ & $76.88 \pm 7.86$ & $76.83 \pm 5.66$ & $73.83 \pm 4.24$ & $F=2.39$ & 0.098 \\
\hline Hypertension $(N, \%)$ & $15(57.69 \%)$ & $19(65.52 \%)$ & $13(44.82 \%)$ & $\chi^{2}=2.69$ & 0.260 \\
\hline $\mathrm{BMI}($ mean $\pm \mathrm{SD})$ & $22.53 \pm 2.47$ & $21.83 \pm 2.60$ & $23.35 \pm 3.02$ & $F=2.29$ & 0.108 \\
\hline Smoking status $(N, \%)$ & $4(15.38 \%)$ & $3(10.34 \%)$ & $1(3.45 \%)$ & $\chi^{2}=2.30$ & 0.316 \\
\hline SGDS-K (mean \pm SD) & $10.15 \pm 3.50$ & $2.65 \pm 2.48$ & $0.52 \pm 0.83$ & $F=111.71$ & 0.000 \\
\hline MMSE-KC (mean \pm SD) & $16.00 \pm 3.46$ & $14.90 \pm 3.56$ & $27.90 \pm 1.59$ & $F=166.00$ & 0.000 \\
\hline $\mathrm{CDR}($ mean $\pm \mathrm{SD})$ & $0.96 \pm 0.37$ & $1.10 \pm 0.60$ & $0.00 \pm 0.00$ & $F=61.32$ & 0.000 \\
\hline
\end{tabular}

Note. AD + depression, Alzheimer's disease with depression; AD - depression, Alzheimer's disease without depression; Control, healthy control; SD, standard deviation; BMI, body mass index; SGDS-K, Korean version of the Geriatric Depression Scale-Short Form; MMSE-KC, Mini-Mental State Examination-Korean version; CDR, Clinical Dementia Rating.

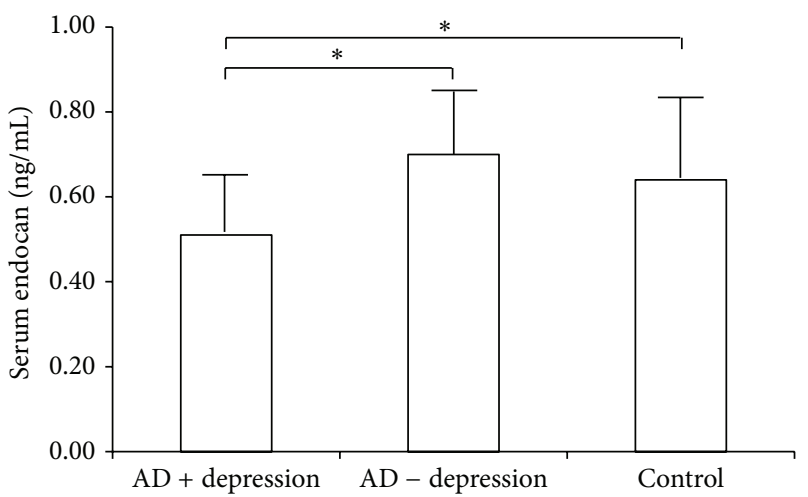

FIGURE 1: Serum endocan levels in $\mathrm{AD}+$ depression $(n=26), \mathrm{AD}-$ depression $(n=29)$, and control $(n=29)$ groups. The $\mathrm{AD}+$ depression group had lower serum endocan levels compared with either of the two groups $(F(2,81)=7.57, p=0.001) .{ }^{*} p<0.05$ on Duncan's post hoc test. The bars indicate standard deviations.

patients $(r=-0.28, p=0.011)$ (Figure 2$)$. There was no significant correlation between endocan levels and the MMSEKC scores $(r=0.02, p=0.878)$.

\section{Discussion}

This study measured, for the first time, serum endocan levels in $\mathrm{AD}$ patients, and these levels were lower in the $\mathrm{AD}+$ depression group than in either the $\mathrm{AD}$ - depression group or the healthy control group. Our analysis showed a negative correlation between serum endocan levels and depressive symptoms in $\mathrm{AD}$, suggesting that serum endocan may be closely related with depressive symptoms in AD.

The underlying mechanism in the relationship between serum endocan levels and depression in $\mathrm{AD}$ is unknown. As a probable mechanism, it can be suggested that increased cortisol levels in depression influence serum endocan levels. It is well known that late-life depression is associated with increased activity of the hypothalamic-pituitary-adrenal (HPA) axis [33], and hypercortisolemia was found in older depressed patients [34]. It was reported that cortisol inhibited

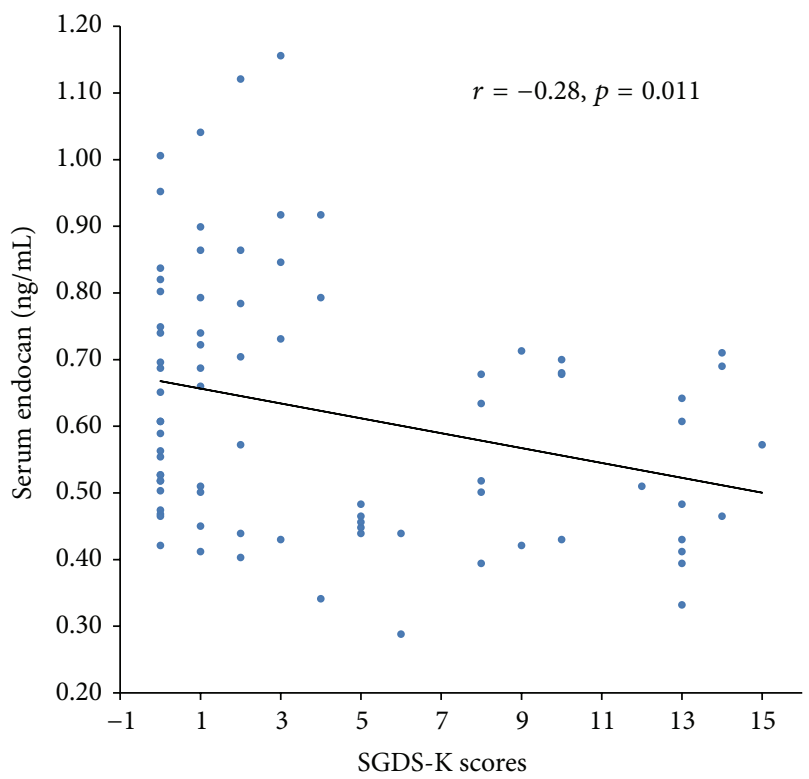

FIGURE 2: Scatter plot for the negative correlation between serum endocan levels and SGDS-K scores $(r=-0.28, p=0.011)$. Note. SGDS-K, Korean version of the Geriatric Depression Scale-Short Form.

endocan gene expression in preadipocytes and endocan production in adipocytes [35]. The results of this current study, based on the relationships outlined above, suggest that serum endocan levels might be decreased due to the inhibitory effect of elevated cortisol on endocan production in $\mathrm{AD}+$ depression group.

Another assumption is that the serum endocan levels might be regulated by inflammatory cytokines that are related to depression. Depressive patients have higher levels of a number of these cytokines, such as tumor necrosis factor$\alpha$ (TNF- $\alpha$ ) [36-38] and interferon- $\gamma$ (IFN- $\gamma$ ) [39-42], and some studies have shown that cytokines such as IFN- $\gamma$ and TNF- $\alpha$ influence endocan expression in endothelial cells. IFN- $\gamma$ inhibited endocan mRNA, which was induced by TNF- $\alpha$ in human umbilical vein endothelial cells (HUVECs). 
When IFN- $\gamma$ was combined with TNF- $\alpha$, endocan mRNA was more strongly inhibited [43]. Another study reported that IFN- $\gamma$ reduced endocan secretion, which was induced by TNF- $\alpha$ in HUVECs [44]. Therefore, in our study, decreased serum endocan levels in the $\mathrm{AD}+$ depression group could be related with the inhibitory effect of IFN- $\gamma$ on endocan levels. Elevated level of IFN- $\gamma$ in depression might reduce endocan secretion. However, we did not directly examine the relationship between endocan and inflammatory cytokines in patients with $\mathrm{AD}+$ depression. Besides, the correlation between endocan and inflammatory cytokines like C-reactive protein (CRP) has also been reported [19, 26, 35]. Additional studies should be conducted to determine this direct relationship and correlation between endocan and inflammatory cytokines in $\mathrm{AD}$ patients with depression.

The clinical significance of decreased serum endocan levels in patients with $\mathrm{AD}+$ depression is unclear. It has been reported that endocan may exert anti-inflammatory and vasculoprotective effects, which may play a role in the process of atherosclerosis [35]. Plasma endocan was decreased in obesity, and it was suggested that low plasma endocan levels may correspond to the loss of the vasoprotective factor in obesity [35]. Previous studies have shown that both vascular impairment $[45,46]$ and inflammation [47-50] can play a role in the progression of $\mathrm{AD}$ and that depression comorbidity in $\mathrm{AD}$ contributes to poor $\mathrm{AD}$ prognosis [51,52]. Therefore, in this study, we reasoned that significantly decreased serum endocan levels in patients with $\mathrm{AD}+$ depression might have reduced the vasoprotective effects or anti-inflammatory activity, which could contribute to poor $\mathrm{AD}$ prognosis. Thus, this finding implicates that endocan might be a biomarker associated with pathophysiology in the $\mathrm{AD}+$ depression and a predictor of disease progression. The pathophysiological mechanisms and role of endocan in patients with $\mathrm{AD}+$ depression should be investigated.

Additionally, in our study, a significant negative correlation was observed between serum endocan levels and depressive symptoms in $\mathrm{AD}$; hence, endocan could be a biomarker for depression severity in $\mathrm{AD}$. It would be of interest to establish whether endocan is related with depressive symptoms in other depressive disorders such as major depression and dysthymic disorder, and thus additional studies on this relationship are also recommended.

This study has some limitations. Healthy control group has a relatively small sample size compared with that of $\mathrm{AD}$ group. Further studies with a larger sample may be needed for generalization of these results. Another limitation is that depressive patients without $\mathrm{AD}$ were not included in the study. To identify whether the presence of depression itself significantly influences the serum endocan levels, additional studies should be performed to examine the relationship between serum endocan and depressive symptoms in the elderly depressed patients. Finally, because this study had a cross-sectional design, we could not evaluate whether serum endocan levels would be altered with improvement of depression. To determine the possibility of endocan as a biomarker (trait or state marker) of depression in $\mathrm{AD}$, studies assessing the serum endocan levels as the depression treatment progresses are needed.

\section{Conclusions}

This is the first study to suggest that serum endocan levels may be associated with depression in AD patients and that endocan might be a biomarker for the severity of depression in AD. This study will promote future studies investigating the pathophysiological mechanisms underlying the role of endocan in patients with $\mathrm{AD}+$ depression or other depressive disorders such as major depression and dysthymic disorder.

\section{Abbreviations \\ $\mathrm{AD}+$ depression: Alzheimer's disease with depression $\mathrm{AD}$ - depression: Alzheimer's disease without depression Control: Healthy control \\ VEGF: Vascular endothelial growth factor \\ ANOVA: One-way analysis of variance \\ SD: $\quad$ Standard deviation \\ BMI: $\quad$ Body mass index \\ MMSE-KC: Mini-Mental State Examination-Korean version \\ SGDS-K: $\quad$ Korean version of the Geriatric \\ CDR: Depression Scale-Short Form \\ TNF- $\alpha$ : $\quad$ Tumor necrosis factor- $\alpha$ \\ IFN- $\gamma$ : Interferon- $\gamma$ \\ HUVECs: Human umbilical vein endothelial cells.}

\section{Conflict of Interests}

The authors declare that there is no conflict of interests regarding the publication of this paper.

\section{Acknowledgment}

This research was supported by Hallym University Research Fund.

\section{References}

[1] A. H. Vagnucci Jr. and W. W. Li, "Alzheimer's disease and angiogenesis," The Lancet, vol. 361, no. 9357, pp. 605-608, 2003.

[2] D. L. Dickstein, J. Walsh, H. Brautigam, S. D. Stockton Jr., S. Gandy, and P. R. Hof, "Role of vascular risk factors and vascular dysfunction in Alzheimer's disease," Mount Sinai Journal of Medicine, vol. 77, no. 1, pp. 82-102, 2010.

[3] E. Tarkowski, R. Issa, M. Sjögren et al., "Increased intrathecal levels of the angiogenic factors VEGF and TGF- $\beta$ in Alzheimer's disease and vascular dementia," Neurobiology of Aging, vol. 23, no. 2, pp. 237-243, 2002.

[4] I. Mateo, J. Llorca, J. Infante et al., "Low serum VEGF levels are associated with Alzheimer's disease," Acta Neurologica Scandinavica, vol. 116, no. 1, pp. 56-58, 2007.

[5] Y. N. Kim and D. H. Kim, "Decreased serum angiogenin level in Alzheimer's disease," Progress in Neuro-Psychopharmacology and Biological Psychiatry, vol. 38, no. 2, pp. 116-120, 2012.

[6] J. Jung, S. Kim, K. Yoon et al., "The effect of depression on serum VEGF level in Alzheimer's disease," Disease Markers, vol. 2015, Article ID 742612, 6 pages, 2015. 
[7] E. Rennel, S. Mellberg, A. Dimberg et al., "Endocan is a VEGF$\mathrm{A}$ and PI3K regulated gene with increased expression in human renal cancer," Experimental Cell Research, vol. 313, no. 7, pp. 1285-1294, 2007.

[8] F. Roudnicky, C. Poyet, P. Wild et al., "Endocan is upregulated on tumor vessels in invasive bladder cancer where it mediates VEGF-A-induced angiogenesis," Cancer Research, vol. 73, no. 3, pp. 1097-1106, 2013.

[9] R. Del Toro, C. Prahst, T. Mathivet et al., "Identification and functional analysis of endothelial tip cell-enriched genes," Blood, vol. 116, no. 19, pp. 4025-4033, 2010.

[10] G. A. Strasser, J. S. Kaminker, and M. Tessier-Lavigne, "Microarray analysis of retinal endothelial tip cells identifies CXCR4 as a mediator of tip cell morphology and branching," Blood, vol. 115, no. 24, pp. 5102-5110, 2010.

[11] L.-Y. Chen, X. Liu, S.-L. Wang, and C.-Y. Qin, “Over-expression of the Endocan gene in endothelial cells from hepatocellular carcinoma is associated with angiogenesis and tumour invasion," Journal of International Medical Research, vol. 38, no. 2, pp. 498-510, 2010.

[12] B. D. Grigoriu, F. Depontieu, A. Scherpereel et al., "Endocan expression and relationship with survival in human non-small cell lung cancer," Clinical Cancer Research, vol. 12, no. 15, pp. 4575-4582, 2006.

[13] X. Leroy, S. Aubert, L. Zini et al., "Vascular endocan (ESM-1) is markedly overexpressed in clear cell renal cell carcinoma," Histopathology, vol. 56, no. 2, pp. 180-187, 2010.

[14] N. Liu, L.-H. Zhang, H. Du et al., "Overexpression of endothelial cell specific molecule-1 (ESM-1) in gastric cancer," Annals of Surgical Oncology, vol. 17, no. 10, pp. 2628-2639, 2010.

[15] A. Scherpereel, T. Gentina, B. Grigoriu et al., "Overexpression of endocan induces tumor formation," Cancer Research, vol. 63, no. 18, pp. 6084-6089, 2003.

[16] H. Jiang, X. Fu, and Y. Chen, "Serum level of endothelial cellspecific molecule-1 and prognosis of colorectal cancer," Genetics and Molecular Research, vol. 14, no. 2, pp. 5519-5526, 2015.

[17] M. M. El Behery, M. A. Seksaka, M. A. Ibrahiem, H. S. Saleh, and Y. El Alfy, "Clinicopathological correlation of endocan expression and survival in epithelial ovarian cancer," Archives of Gynecology and Obstetrics, vol. 288, no. 6, pp. 1371-1376, 2013.

[18] M. I. Yilmaz, D. Siriopol, M. Saglam et al., "Plasma endocan levels associate with inflammation, vascular abnormalities, cardiovascular events, and survival in chronic kidney disease," Kidney International, vol. 86, no. 6, pp. 1213-1220, 2014.

[19] S. Balta, D. P. Mikhailidis, S. Demirkol et al., "Endocan-a novel inflammatory indicator in newly diagnosed patients with hypertension: a pilot study," Angiology, vol. 65, no. 9, pp. 773$777,2014$.

[20] S. Balta, D. P. Mikhailidis, S. Demirkol, C. Ozturk, T. Celik, and A. Iyisoy, "Endocan: a novel inflammatory indicator in cardiovascular disease?" Atherosclerosis, vol. 243, no. 1, pp. 339343, 2015.

[21] M. Kose, S. Emet, T. S. Akpinar et al., "Serum endocan level and the severity of coronary artery disease: a pilot study," Angiology, vol. 66, no. 8, pp. 727-731, 2015.

[22] S. Balta, D. P. Mikhailidis, S. Demirkol, T. Celik, C. Ozturk, and A. Iyisoy, "Endocan and atherosclerosis," Angiology, vol. 66, no. 5, p. 490, 2015.

[23] C. Xiong, Z.-W. Zhao, Z.-Y. Chen et al., "Elevated human endothelial cell-specific molecule-1 level and its association with coronary artery disease in patients with hypertension," Journal of Investigative Medicine, vol. 63, no. 7, pp. 867-870, 2015.
[24] A. Scherpereel, F. Depontieu, B. Grigoriu et al., "Endocan, a new endothelial marker in human sepsis," Critical Care Medicine, vol. 34, no. 2, pp. 532-537, 2006.

[25] I. Balta, S. Balta, S. Demirkol et al., "Elevated serum levels of endocan in patients with psoriasis vulgaris: correlations with cardiovascular risk and activity of disease," British Journal of Dermatology, vol. 169, no. 5, pp. 1066-1070, 2013.

[26] I. Balta, S. Balta, O. M. Koryurek et al., "Serum endocan levels as a marker of disease activity in patients with Behçet disease," Journal of the American Academy of Dermatology, vol. 70, no. 2, pp. 291-296, 2014.

[27] K. F. Rodrigues, N. T. Pietrani, A. A. Bosco et al., "Endocan: a new biomarker associated with inflammation in type 2 diabetes mellitus?" Diabetes/Metabolism Research and Reviews, vol. 31, no. 5, pp. 479-480, 2015.

[28] D. Pauly, S. Hamed, M. Behnes et al., "Endothelial cellspecific molecule-1/endocan: diagnostic and prognostic value in patients suffering from severe sepsis and septic shock," Journal of Critical Care, vol. 31, no. 1, pp. 68-75, 2016.

[29] J. T. Olin, L. S. Schneider, I. R. Katz et al., "Provisional diagnostic criteria for depression of Alzheimer disease," The American Journal of Geriatric Psychiatry, vol. 10, no. 2, pp. 125-128, 2002.

[30] D. Y. Lee, K. U. Lee, J. H. Lee et al., "A normative study of the mini-mental state examination in the Korean elderly," Journal of Korean Neuropsychiatric Association, vol. 41, no. 3, pp. 508-525, 2002.

[31] C. P. Hughes, L. Berg, and W. L. Danziger, "A new clinical scale for the staging of dementia," The British Journal of Psychiatry, vol. 140, no. 6, pp. 566-572, 1982.

[32] J. N. Bae and M. J. Cho, "Development of the Korean version of the Geriatric Depression Scale and its short form among elderly psychiatric patients," Journal of Psychosomatic Research, vol. 57, no. 3, pp. 297-305, 2004.

[33] D. Dobie and M. Raskind, "Biology in geriatric psychiatry: diagnostic implications," in Review of Psychiatry, vol. 9, pp. 232249, American Psychiatric Press, Washington, DC, USA, 1990.

[34] I. J. E. Heuser, U. Schweiger, U. Gotthardt et al., "Pituitaryadrenal-system regulation and psychopathology during amitriptyline treatment in elderly depressed patients and normal comparison subjects," The American Journal of Psychiatry, vol. 153, no. 1, pp. 93-99, 1996.

[35] J. Janke, S. Engeli, K. Gorzelniak et al., "Adipose tissue and circulating endothelial cell specific molecule-1 in human obesity," Hormone and Metabolic Research, vol. 38, no. 1, pp. 28-33, 2006.

[36] S. Lanquillon, J.-C. Krieg, U. Bening-Abu-Shach, and H. Vedder, "Cytokine production and treatment response in major depressive disorder," Neuropsychopharmacology, vol. 22, no. 4, pp. 370-379, 2000.

[37] O. Mikova, R. Yakimova, E. Bosmans, G. Kenis, and M. Maes, "Increased serum tumor necrosis factor alpha concentrations in major depression and multiple sclerosis," European Neuropsychopharmacology, vol. 11, no. 3, pp. 203-208, 2001.

[38] G. L. Xiong, K. Prybol, S. H. Boyle et al., "Inflammation markers and major depressive disorder in patients with chronic heart failure: results from the sertraline against depression and heart disease in chronic heart failure study," Psychosomatic Medicine, vol. 77, no. 7, pp. 808-815, 2015.

[39] V. Gabbay, R. G. Klein, C. M. Alonso et al., "Immune system dysregulation in adolescent major depressive disorder," Journal of Affective Disorders, vol. 115, no. 1-2, pp. 177-182, 2009. 
[40] V. Gabbay, R. G. Klein, L. E. Guttman et al., "A preliminary study of cytokines in suicidal and nonsuicidal adolescents with major depression," Journal of Child and Adolescent Psychopharmacology, vol. 19, no. 4, pp. 423-430, 2009.

[41] F. M. Schmidt, N. Lichtblau, J. Minkwitz et al., "Cytokine levels in depressed and non-depressed subjects, and masking effects of obesity," Journal of Psychiatric Research, vol. 55, no. 1, pp. 29-34, 2014.

[42] N. M. Simon, K. McNamara, C. W. Chow et al., "A detailed examination of cytokine abnormalities in Major Depressive Disorder," European Neuropsychopharmacology, vol. 18, no. 3, pp. 230-233, 2008.

[43] P. Lassalle, S. Molet, A. Janin et al., "ESM-1 is a novel human endothelial cell-specific molecule expressed in lung and regulated by cytokines," The Journal of Biological Chemistry, vol. 271, no. 34, pp. 20458-20464, 1996.

[44] D. Bechard, V. Meignin, A. Scherpereel et al., "Characterization of the secreted form of endothelial-cell-specific molecule 1 by specific monoclonal antibodies," Journal of Vascular Research, vol. 37, no. 5, pp. 417-425, 2000.

[45] K. A. Jellinger and J. Attems, "Incidence of cerebrovascular lesions in Alzheimer's disease: a postmortem study," Acta Neuropathologica, vol. 105, no. 1, pp. 14-17, 2003.

[46] B. V.Zlokovic, "Neurovascular mechanisms of Alzheimer's neurodegeneration," Trends in Neurosciences, vol. 28, no. 4, pp. 202208, 2005.

[47] H. Akiyama, S. Barger, S. Barnum et al., "Inflammation and Alzheimer's disease," Neurobiology of Aging, vol. 21, no. 3, pp. 383-421, 2000.

[48] P. Grammas and R. Ovase, "Inflammatory factors are elevated in brain microvessels in Alzheimer's disease," Neurobiology of Aging, vol. 22, no. 6, pp. 837-842, 2001.

[49] M. T. Heneka and M. K. O'Banion, "Inflammatory processes in Alzheimer's disease," Journal of Neuroimmunology, vol. 184, no. 1-2, pp. 69-91, 2007.

[50] T. Wyss-Coray, "Inflammation in Alzheimer disease: driving force, bystander or beneficial response?" Nature Medicine, vol. 12, no. 9, pp. 1005-1015, 2006.

[51] M. A. Rapp, M. Schnaider-Beeri, D. P. Purohit, D. P. Perl, V. Haroutunian, and M. Sano, "Increased neurofibrillary tangles in patients with Alzheimer disease with comorbid depression," The American Journal of Geriatric Psychiatry, vol. 16, no. 2, pp. 168-174, 2008.

[52] G. Spalletta, C. Caltagirone, P. Girardi, W. Gianni, A. R. Casini, and K. Palmer, "The role of persistent and incident major depression on rate of cognitive deterioration in newly diagnosed Alzheimer's disease patients," Psychiatry Research, vol. 198, no. 2, pp. 263-268, 2012. 


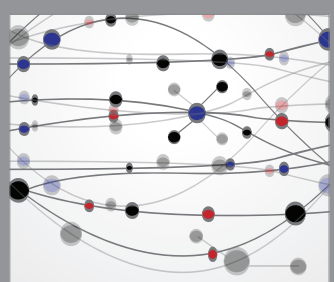

The Scientific World Journal
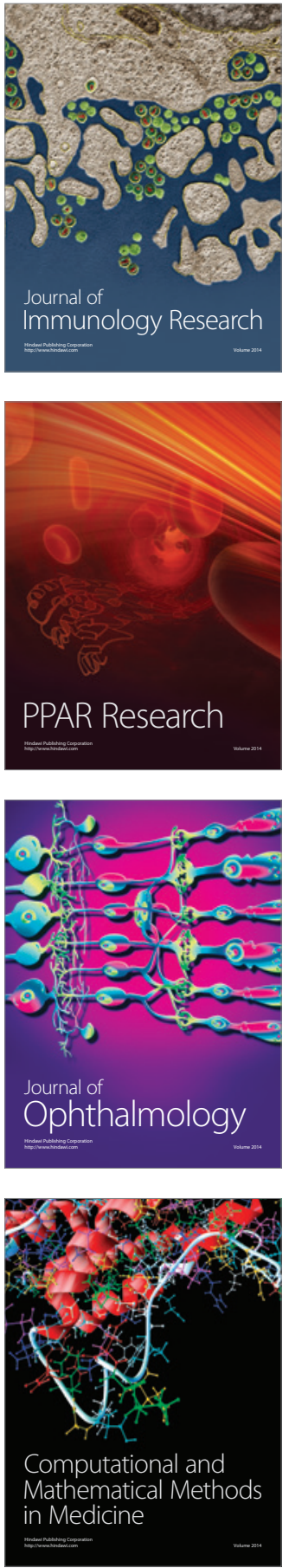

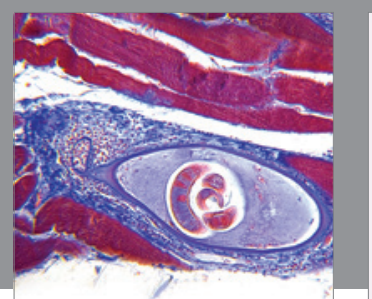

Gastroenterology Research and Practice

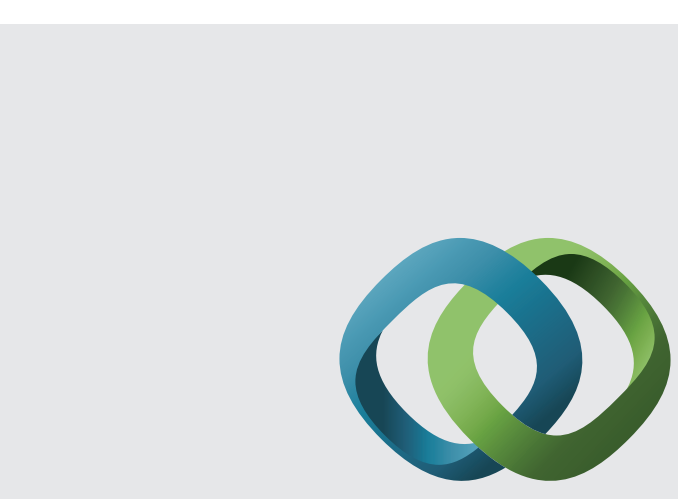

\section{Hindawi}

Submit your manuscripts at

http://www.hindawi.com
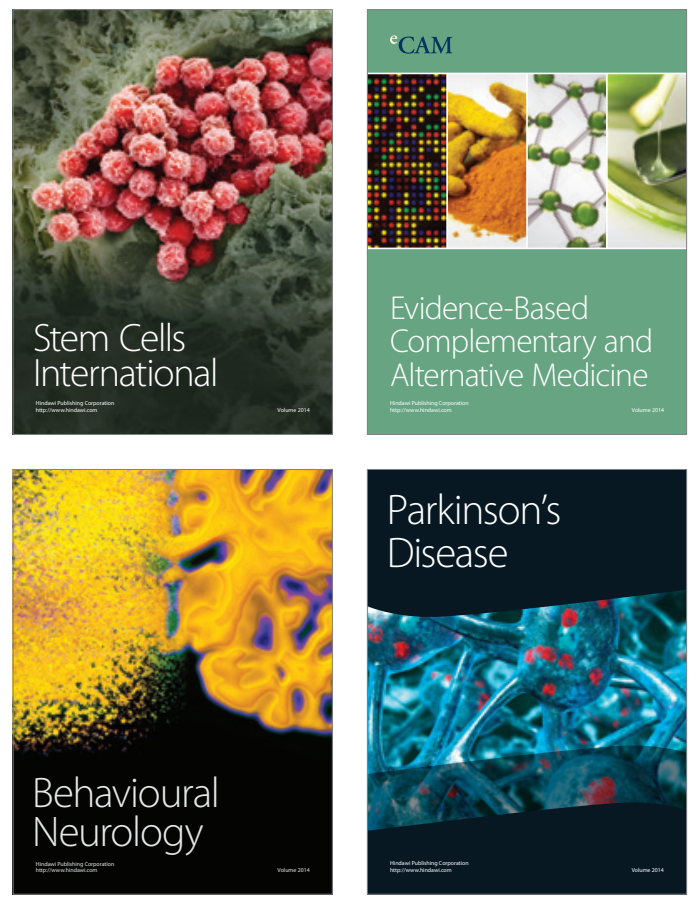
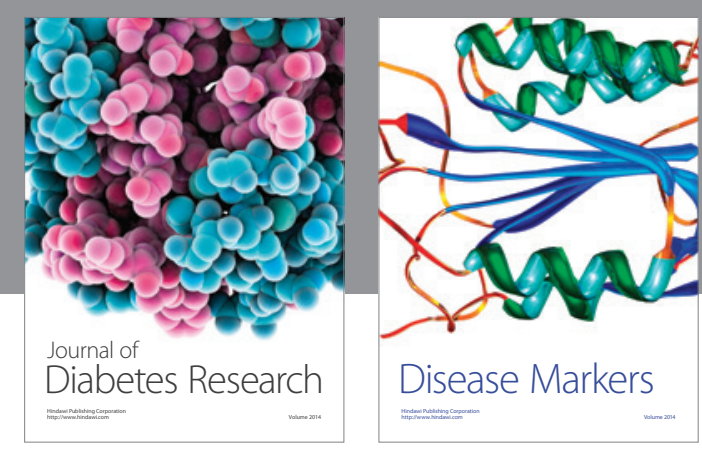

Disease Markers
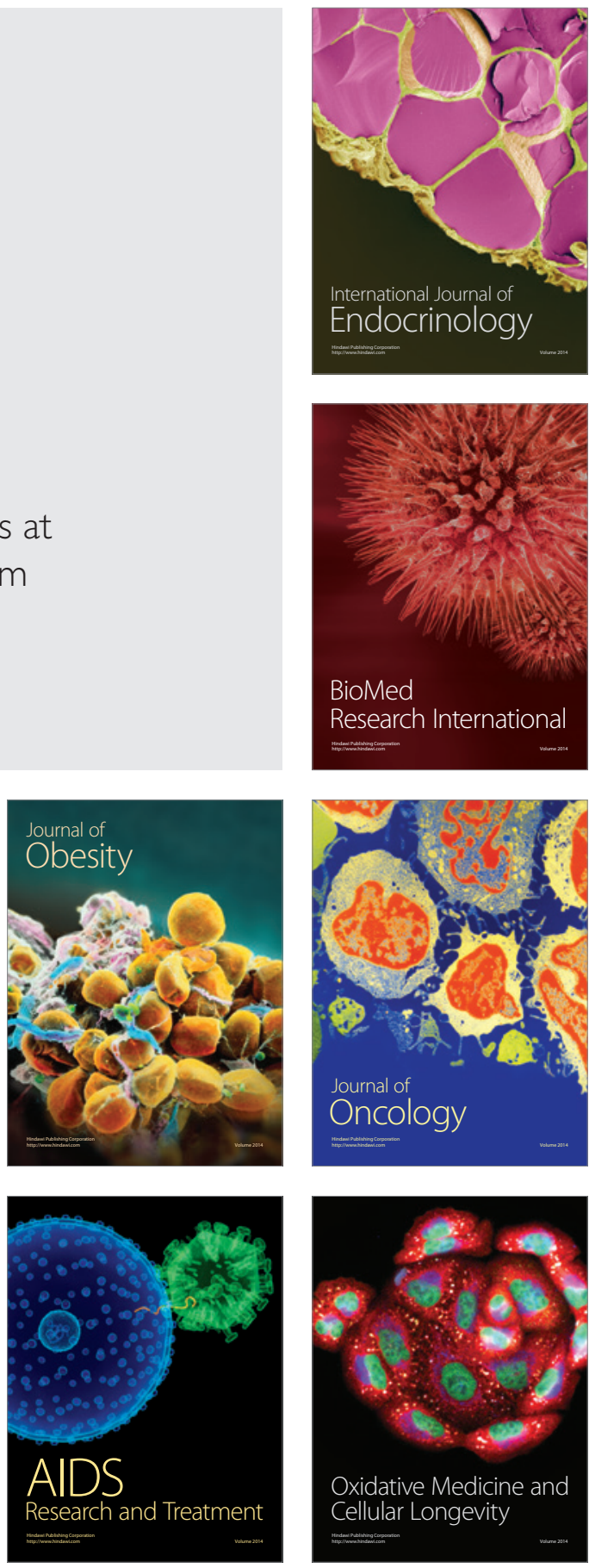